\title{
Pacific
}

Journal of

Mathematics

\section{THE TURAEV AND THURSTON NORMS}

Stefan Friedl, Daniel S. Silver and Susan G. Williams 


\title{
THE TURAEV AND THURSTON NORMS
}

\author{
Stefan Friedl, Daniel S. Silver and Susan G. Williams
}

\begin{abstract}
In 1986, W. Thurston introduced a (possibly degenerate) norm on the first cohomology group of a 3-manifold. Inspired by this definition, Turaev introduced in $\mathbf{2 0 0 2}$ an analogous norm on the first cohomology group of a finite 2-complex. We show that if $N$ is the exterior of a link in a rational homology sphere, then the Thurston norm agrees with a suitable variation of Turaev's norm defined on any 2-skeleton of $N$.
\end{abstract}

\section{Introduction}

W. Thurston [1986] introduced a seminorm for 3-manifolds $N$ with empty or toroidal boundary. It is a function $x_{N}: H^{1}(N ; \mathbb{Q}) \rightarrow \mathbb{Q}_{\geq 0}$ which measures the complexity of surfaces that are dual to cohomology classes. We adopt the custom of referring to $x_{N}$ as the Thurston norm. It plays a central role in 3-manifold topology and we recall its definition in Section 2A, where we will also review several of its key properties.

Later, V. Turaev [2002] introduced an analogously defined seminorm for 2complexes. For any finite 2-complex $X$ with suitably defined boundary $\partial X$, Turaev defined $t_{X}: H^{1}(X, \partial X ; \mathbb{Q}) \rightarrow \mathbb{Q}_{\geq 0}$ using complexities of dual 1-complexes. Inspired by work of C. McMullen [2002], Turaev gave lower bounds for $t_{X}$ in terms of the multivariable Alexander polynomial whenever the boundary of $X$ is empty. The precise definition of $\partial X$ will be recalled in Section 2B. For the purpose of the introduction it suffices to know that if $N$ is a compact triangulated 3-manifold, then the 2-skeleton $N^{(2)}$ is a finite 2-complex with empty boundary.

A homotopy equivalence induces a canonical isomorphism of homology and cohomology groups which we use to identify the groups. Examples given in [Turaev 2002, p. 143] show that $t_{X}$ is not invariant under homotopy. We therefore introduce the following variation: For any finite 2-complex $X$ with empty boundary, we define the Turaev complexity function as follows. If $\phi \in H^{1}(X ; \mathbb{Q})=\operatorname{Hom}\left(\pi_{1}(X), \mathbb{Q}\right)$, then

$$
\bar{t}_{X}(\phi):=\inf \left\{\begin{array}{l|l}
t_{Y}(\phi \circ f) & \begin{array}{l}
Y \text { is a finite 2-complex with } \partial Y=\varnothing \text { and } \\
f: \pi_{1}(Y) \rightarrow \pi_{1}(X) \text { is an isomorphism }
\end{array}
\end{array}\right\} .
$$

MSC2010: 57M27.

Keywords: Turaev norm, Thurston norm, links, Alexander polynomials. 
Clearly $\bar{t}_{X}$ depends only on the fundamental group of $X$. Since the minimum of two norms need not satisfy the triangle inequality, the Turaev function is not a seminorm, as we will see later in Proposition 4.2.

For any 3-manifold $N$, we further define

$$
\bar{t}_{N}(\phi):=\bar{t}_{N^{(2)}}(\phi)
$$

where $N^{(2)}$ is the 2-skeleton of a triangulation of $N$. It is clear from the definition of $\bar{t}$ that $\bar{t}_{N}$ does not depend on the choice of a triangulation.

Given a 3-manifold $N$, it is natural to compare $x_{N}$ and $\bar{t}_{N}$ on $H^{1}(N ; \mathbb{Q})$. In general, they do not agree. Indeed in Section 4A we will see that there exist many examples of closed 3-manifolds $N$ and classes $\phi \in H^{1}(N ; \mathbb{Z})$ such that $\bar{t}_{N}(\phi)>x_{N}(\phi)$. The underlying reason is quite obvious: the Thurston norm is defined using complexities of surfaces, whereas the Turaev function is defined using complexities of graphs. However, the complexity of a closed surface is lower by at least one than the complexity of any underlying 1-skeleton.

It is therefore reasonable to restrict ourselves to the class of 3-manifolds where Thurston norm-minimizing surfaces can always be chosen to have no closed component. In Lemma 4.5 we will see that if $N=\Sigma^{3} \backslash v L$ is the exterior of a of a link $L$ in a rational homology sphere $\Sigma$, then $N$ has this property. For simplicity of exposition we henceforth restrict ourselves to this type of 3-manifolds.

Using explicit and elementary constructions of 2-complexes, we prove the following.

Theorem 1.1. Let $N$ be the exterior of a link in a rational homology sphere. Then

$$
\bar{t}_{N}(\phi) \leq x_{N}(\phi) \quad \text { for any } \phi \in H^{1}(N ; \mathbb{Q}) .
$$

It is natural to ask whether the extra freedom provided by working with 2complexes instead of 3-manifolds allows us to get lower complexities. Our main theorem says that this is not the case, at least if we restrict ourselves to irreducible link exteriors. (Note that it follows from the definitions and from Schönflies theorem that the exterior of a link $L$ in $S^{3}$ is irreducible if and only if $L$ is nonsplit.)

Theorem 1.2. Let $N$ be the exterior of a link in a rational homology sphere. If $N$ is irreducible, then

$$
\bar{t}_{N}(\phi)=x_{N}(\phi) \quad \text { for any } \phi \in H^{1}(N ; \mathbb{Q}) .
$$

We will prove the inequality $\bar{t}_{X}(\phi) \geq x_{N}(\phi)$ by studying the Alexander norms of finite covers of $X$ and $N$, and by applying the recent results of I. Agol [2008; 2013], D. Wise [2009; 2012b; 2012a], P. Przytycki and D. Wise [2014; 2012] and Y. Liu [2013]. We do not know of an elementary proof of Theorem 1.2. 
Theorem 1.2 fits into a long sequence of results showing that minimal-genus Seifert surfaces and Thurston norm-minimizing surfaces are "robust" in the sense that they "stay minimal" even if one relaxes some conditions. Examples of this phenomenon have been found by many authors, see, for example, [Gabai 1983; 1987; Kronheimer 1999; Friedl and Vidussi 2014; Nagel 2014; Friedl et al. 2015].

The paper is organized as follows. In Section 2 we recall the definition of the Thurston and Turaev norms, and we introduce the Turaev complexity function. In Section 3 we discuss the Alexander norm for 3-manifolds and 2-complexes, and we recall how they give lower bounds on the Thurston norm and Turaev complexity function, respectively. In Section 4A, we first show that the Turaev complexity function of the 2-skeleton can be greater than the corresponding Thurston norm. We then show in Section 4B that the Thurston norm of any irreducible 3-manifold with nontrivial toroidal boundary is detected by the Alexander norm of an appropriate finite cover. Finally, in Section 4C we put everything together to prove Theorem 1.2.

Conventions. All 3-manifolds are compact, orientable and connected, and all 2complexes are connected, unless it says specifically otherwise.

\section{The definition of the Thurston norm and the Turaev norm}

2A. The Thurston norm and fibered classes. Let $N$ be a 3-manifold with empty or toroidal boundary. The Thurston norm of a class $\phi \in H^{1}(N ; \mathbb{Z})$ is defined as

$$
x_{N}(\phi)=\min \left\{\chi_{-}(\Sigma) \mid \Sigma \subset N \text { properly embedded surface dual to } \phi\right\} .
$$

Here, $\chi_{-}(\Sigma)$ is the complexity of a surface $\Sigma$ with connected components

$$
\Sigma_{1}, \ldots, \Sigma_{k}
$$

given by

$$
\chi_{-}(\Sigma)=\sum_{i=1}^{k} \max \left\{-\chi\left(\Sigma_{i}\right), 0\right\} .
$$

Thurston [1986] showed that $x_{N}$ defines a (possibly degenerate) norm on $H^{1}(N ; \mathbb{Z})$. Note that any norm on $H^{1}(N ; \mathbb{Z})$ extends uniquely to a norm on $H^{1}(N ; \mathbb{Q})$, which we denote by the same symbol.

We say that a class $\phi \in H^{1}(N ; \mathbb{Q})$ is fibered if there exists a fibration $p: N \rightarrow S^{1}$ such that $\phi$ lies in the pull-back of $H^{1}\left(S^{1} ; \mathbb{Q}\right)$ under $p$. By [Tischler 1970], a class $\phi \in H^{1}(N ; \mathbb{Q})$ is fibered if and only if it can be represented by a nondegenerate closed 1-form.

Thurston [1986] showed the Thurston norm ball

$$
\left\{\phi \in H^{1}(N ; \mathbb{Q}) \mid x_{N}(\phi) \leq 1\right\}
$$


is a polyhedron. This implies that if $C$ is a cone on a face of the polyhedron, then the restriction of $x_{N}$ to $C$ is a linear function. To put differently, for any $\alpha, \beta \in C$ and nonnegative $r, s \in \mathbb{Q}_{\geq 0}$, the linear combination $r \alpha+s \beta$ also lies in $C$, and $x_{N}(r \alpha+s \beta)=r x_{N}(\alpha)+s x_{N}(\beta)$.

Thurston [1986] also showed that any fibered class lies in the open cone on a top-dimensional face of the Thurston norm ball. Furthermore, any other class in that open cone is also fibered. Consequently, the set of fibered classes is the union of open cones on top-dimensional faces of the Thurston norm ball. We will refer to these cones as the fibered cones of $N$. A class $\phi \in H^{1}(N ; \mathbb{Q})$ in the closure of a fibered cone is quasifibered.

2B. The Turaev norm and the Turaev complexity function for 2-complexes. As in [Turaev 2002], a finite 2-complex is the underlying topological space of a finite connected 2-dimensional $\mathrm{CW}$-complex such that each point has a neighborhood homeomorphic to the cone over a finite graph. Examples of finite 2-complexes are given by compact surfaces (see [Turaev 2002, p. 138]), 2-skeletons of finite simplicial spaces, and the products of graphs with a closed interval.

The interior of $X$, denoted Int $X$, is the set of points in $X$ that have neighborhoods homeomorphic to $\mathbb{R}^{2}$. Finally the boundary $\partial X$ of $X$ is the closure in $X$ of the set of all points of $X \backslash$ Int $X$ that have open neighborhoods in $X$ homeomorphic to $\mathbb{R}$ or to $\mathbb{R} \times \mathbb{R}_{\geq 0}$. Note that $\partial X$ is a graph contained in the 1-skeleton of the CWdecomposition of $X$. For example, if $X$ is a compact surface, then $\partial X$ is precisely the boundary of $X$ in the usual sense.

Following [Turaev 2002], we say that a graph $\Gamma$ in a finite 2-complex is regular if $\Gamma \subset X \backslash \partial X$ and if there exists a closed neighborhood in $X \backslash \partial X$ homeomorphic to $\Gamma \times[-1,1]$ so that $\Gamma=\Gamma \times 0$. A coorientation for a regular graph $\Gamma$ with components $\Gamma_{1}, \ldots, \Gamma_{k}$ is the choice of a component of $\Gamma_{i} \times[-1,1] \backslash \Gamma_{i}$, for each $i=1, \ldots, k$. A cooriented regular graph $\Gamma \subset X$ canonically defines an element $\phi_{\Gamma} \in H^{1}(X, \partial X ; \mathbb{Z})$. Given any $\phi \in H^{1}(X, \partial X ; \mathbb{Z})$, there exists a cooriented regular graph $\Gamma$ with $\phi_{\Gamma}=\phi$. (We refer to [Turaev 2002] for details.)

Let $X$ be a finite 2-complex with $\partial X=\varnothing$, and let $\phi \in H^{1}(X ; \mathbb{Z})$. The Turaev norm of $\phi$ is

$$
t_{X}(\phi):=\min \left\{\chi_{-}(\Gamma) \mid \Gamma \subset X \text { cooriented regular graph with } \phi_{\Gamma}=\phi\right\},
$$

where $\chi_{-}(\Gamma)$ is the complexity of a graph $\Gamma$ with connected components $\Gamma_{1}, \ldots, \Gamma_{k}$, given by

$$
\chi_{-}(\Gamma):=\sum_{i=1}^{k} \max \left\{-\chi\left(\Gamma_{i}\right), 0\right\}
$$


Turaev [2002] showed that $t_{X}: H^{1}(X ; \mathbb{Z}) \rightarrow \mathbb{Z}_{\geq 0}$ is a (possibly degenerate) norm, and, as in the previous section, $t_{X}$ extends to a norm

$$
t_{X}: H^{1}(X ; \mathbb{Q}) \rightarrow \mathbb{Q}_{\geq 0} .
$$

In Theorem 5.1 we will show that in general one has to allow disconnected graphs $\Gamma$ to minimize the Turaev norm.

As we already mentioned in the introduction, Turaev [2002, p. 143] showed that $t_{X}$ is in general not invariant under homotopy equivalence. (In fact Turaev showed that $t_{X}$ is not even invariant under simple homotopy.) We therefore introduce a variation of the Turaev norm: if $X$ is a finite 2-complex with $\partial X=\varnothing$, then given $\phi \in H^{1}(X ; \mathbb{Q})=\operatorname{Hom}\left(\pi_{1}(X), \mathbb{Q}\right)$ the Turaev complexity function of $\phi$ is

$$
\bar{t}_{X}(\phi):=\inf \left\{\begin{array}{l|l}
t_{\Gamma}(\phi \circ f) & \begin{array}{l}
\Gamma \text { is a finite 2-complex with } \partial \Gamma=\varnothing \text { and } \\
f: \pi_{1}(\Gamma) \rightarrow \pi_{1}(X) \text { is an isomorphism }
\end{array}
\end{array}\right\} .
$$

We make the following observations:

(i) It is clear that $\bar{t}_{X}$ is invariant under homotopy equivalence. In fact $\bar{t}_{X}$ depends only on the fundamental group of $X$.

(ii) Since $\bar{t}_{X}$ is the infimum of continuous homogeneous functions (i.e., functions with $f(\lambda x)=\lambda f(x)$ for $\lambda>0), \bar{t}_{X}$ is upper semicontinuous and homogeneous.

(iii) The complexity function $\bar{t}_{X}$ is defined as the infimum of norms. Note that the minimum of two norms is in general no longer a norm. For example, the infimum of the two norms $a(x, y):=|x|$ and $b(x, y):=|y|$ on $\mathbb{R}^{2}$ is not a norm. We will see in Proposition 4.2 that $\bar{t}_{X}(\phi)$ is, in general, not a norm.

(iv) From the definition, it follows immediately that

$$
\bar{t}_{X}(\phi) \leq t_{X}(\phi),
$$

for any $\phi \in H^{1}(X ; \mathbb{Q})$.

(v) For any finite 2-complex $X$, Turaev [2002, Section 1.6] shows that $t_{X}$ is algorithmically computable. We do not know whether this is also the case for the Turaev complexity function $\bar{t}_{X}$.

2C. An inequality between the Thurston norm and the Turaev complexity function. The goal of this section is to prove the following inequality between the Thurston norm and the Turaev complexity function.

Proposition 2.1. Let $N$ be a 3-manifold and let $\phi \in H^{1}(N ; \mathbb{Z})$. If $\phi$ is dual to a properly embedded Thurston norm minimizing surface with $r$ closed components, then

$$
\bar{t}_{N}(\phi) \leq x_{N}(\phi)+r .
$$

Proof. Let $\phi \in H^{1}(N ; \mathbb{Z})$ and let $\Sigma=\Sigma_{1} \cup \cdots \cup \Sigma_{s}$ be a surface dual to $\phi$ 
of minimal complexity such that $\Sigma_{1}, \ldots, \Sigma_{r}$ are closed and $\Sigma_{r+1}, \ldots, \Sigma_{s}$ have nonempty boundary.

For $i=1, \ldots, r$ we pick an embedded graph $\Gamma_{i} \subset \Sigma_{i}$ with $\chi\left(\Gamma_{i}\right)=\chi\left(\Sigma_{i}\right)-1$ and such that $\pi_{1}\left(\Gamma_{i}\right)$ surjects onto $\pi_{1}\left(\Sigma_{i}\right)$. Furthermore, for $i=r+1, \ldots, s$ we pick an embedded graph $\Gamma_{i} \subset \Sigma_{i}$ with $\chi\left(\Gamma_{i}\right)=\chi\left(\Sigma_{i}\right)$ and such that $\pi_{1}\left(\Gamma_{i}\right)$ surjects onto $\pi_{1}\left(\Sigma_{i}\right)$.

Next we select pairwise disjoint product neighborhoods

$$
\Sigma_{1} \times[-1,1], \ldots, \Sigma_{s} \times[-1,1]
$$

such that the product orientations match the orientation of $N$. We equip

$$
M:=N \backslash \bigcup_{i=1}^{s} \Sigma_{i} \times(-1,1)
$$

with a triangulation such that each $\Gamma_{i} \times\{ \pm 1\}$ is a subspace of $M^{(1)}$. Consider

$$
Y:=M^{(2)} \cup \bigcup_{i=1}^{s} \Gamma_{i} \times(-1,1) .
$$

It is straightforward to see that $Y$ is a finite 2-complex with $\partial Y=\varnothing$, and the inclusion map $Y \rightarrow N$ induces an isomorphism of fundamental groups. By slight abuse of notation we denote the restriction of $\phi$ to $Y$ again by $\phi$.

For $i=1, \ldots, s$, we identify $\Gamma_{i}$ with $\Gamma_{i} \times 0$. It is clear that $\Gamma:=\Gamma_{1} \cup \cdots \cup \Gamma_{s}$ is a regular graph on $Y$. Furthermore, with the obvious coorientation, we have $\phi_{\Gamma}=\phi$. It follows that

$$
\begin{aligned}
\bar{t}_{N}(\phi) \leq t_{Y}(\phi) \leq \chi_{-}(\Gamma) & =\sum_{i=1}^{r} \max \left\{-\chi\left(\Gamma_{i}\right), 0\right\}+\sum_{i=r+1}^{s} \max \left\{-\chi\left(\Gamma_{i}\right), 0\right\} \\
& \leq \sum_{i=1}^{r} \max \left\{-\chi\left(\Sigma_{i}\right)+1,0\right\}+\sum_{i=r+1}^{s} \max \left\{-\chi\left(\Sigma_{i}\right), 0\right\} \\
& \leq \chi_{-}(\Sigma)+r \\
& =x_{N}(\phi)+r .
\end{aligned}
$$

Theorem 1.1. Let $N$ be the exterior of a link in a rational homology sphere. Then for any $\phi \in H^{1}(N ; \mathbb{Q})$, we have

$$
\bar{t}_{N}(\phi) \leq x_{N}(\phi) .
$$

Proof. Let $N$ be the exterior of a link in a rational homology sphere. We write $X=N^{(2)}$. Since $\bar{t}$ and $x_{N}$ are homogeneous, it suffices to show that $\bar{t}_{X}(\phi) \leq x_{N}(\phi)$ for every $\phi \in H^{1}(N ; \mathbb{Z})$. Assume that $\phi \in H^{1}(N ; \mathbb{Z})$. By Lemma 4.5 (see 
Section 4A) there exists a Thurston norm-minimizing surface dual to $\phi$ such that each component has nonempty boundary. The desired inequality follows immediately from Proposition 2.1.

\section{Lower bounds on the norms coming from Alexander polynomials}

3A. The Alexander polynomial. Let $X$ be a compact CW-complex, and let

$$
\varphi: H_{1}(X ; \mathbb{Z}) \rightarrow H
$$

be a homomorphism onto a free abelian group. We denote by $\tilde{X}^{\varphi}$ the cover of $X$ corresponding to $\varphi: \pi_{1}(X) \rightarrow H_{1}(X ; \mathbb{Z}) \rightarrow H$. The group $H$ is the deck transformation group of $\tilde{X}^{\varphi} \rightarrow X$, and it acts on $H_{1}\left(\tilde{X}^{\varphi} ; \mathbb{Z}\right)$. Thus we can view $H_{1}\left(\tilde{X}^{\varphi} ; \mathbb{Z}\right)$ as a $\mathbb{Z}[H]$-module. Since $\mathbb{Z}[H]$ is a Noetherian ring, it follows that $H_{1}\left(\tilde{X}^{\varphi} ; \mathbb{Z}\right)$ is a finitely presented $\mathbb{Z}[H]$-module. This means that there exists an exact sequence

$$
\mathbb{Z}[H]^{r} \stackrel{A}{\rightarrow} \mathbb{Z}[H]^{s} \rightarrow H_{1}\left(\tilde{X}^{\varphi} ; \mathbb{Z}\right) \rightarrow 0 .
$$

After possibly adding columns of zeros, we can assume that $r \geq s$. Define the Alexander polynomial of $(X, \varphi)$ to be

$$
\Delta_{X, \varphi}:=\operatorname{gcd} \text { of all } s \times s \text {-minors of } A .
$$

We refer to [Fox 1954; Turaev 2001; Hillman 2012] for the proof of the classical fact that $\Delta_{X, \varphi}$ is well-defined up to multiplication by a unit in $\mathbb{Z}[H]$, i.e., up to multiplication by an element of the form $\epsilon h$, where $\epsilon \in\{-1,1\}$ and $h \in H$.

If $\varphi: H_{1}(X ; \mathbb{Z}) \rightarrow H:=H_{1}(X ; \mathbb{Z}) /$ torsion is the canonical projection, then we write $\Delta_{X}:=\Delta_{X, \varphi}$, and we refer to it as the Alexander polynomial $\Delta_{X}$ of $X$. Furthermore, if $\phi \in H^{1}(X ; \mathbb{Z})=\operatorname{Hom}\left(\pi_{1}(X), \mathbb{Z}\right)$, then we view the corresponding Alexander polynomial $\Delta_{X, \varphi}$ as an element in $\mathbb{Z}\left[t^{ \pm 1}\right]$ under the canonical identification of the group ring $\mathbb{Z}[\mathbb{Z}]$ with the Laurent polynomial ring $\mathbb{Z}\left[t^{ \pm 1}\right]$.

3B. The one-variable Alexander polynomials. In this section we relate the degrees of one-variable Alexander polynomials to the Thurston norm and to the Turaev complexity function.

In the following, given a nonzero polynomial $p(t)=\sum_{i=r}^{s} a_{i} t^{i}$ with $a_{r} \neq 0$ and $a_{s} \neq 0$, we write

$$
\operatorname{deg}(p(t))=s-r .
$$

Note that the degree of a nonzero one-variable Alexander polynomial is well-defined.

The following proposition is well known, see, for example, [Friedl and Kim 2006] for a proof. 
Proposition 3.1. Let $N$ be a closed 3-manifold and let $\phi \in H^{1}(N ; \mathbb{Z})$ be primitive. If $\Delta_{N, \phi} \neq 0$, then

$$
x_{N}(\phi) \geq \operatorname{deg}\left(\Delta_{N, \phi}\right)-2 .
$$

Furthermore, equality holds if $\phi$ is a fibered class and if $N \neq S^{1} \times S^{2}$.

We prove the following.

Proposition 3.2. Let $X$ be a finite 2-complex with $\partial X=\varnothing$, and let be $\phi \in H^{1}(N ; \mathbb{Z})$ primitive. If $\Delta_{X, \phi} \neq 0$, then

$$
\bar{t}_{X}(\phi) \geq \operatorname{deg}\left(\Delta_{X, \phi}\right)-1 \text {. }
$$

Proof. Let $Y$ be a finite 2-complex with $\partial Y=\varnothing$, and let $\psi \in H^{1}(Y ; \mathbb{Z})$ be primitive. If $\Delta_{Y, \phi} \neq 0$, then it follows from Claim 2 of [Turaev 2002, p. 152] that

$$
t_{Y}(\psi) \geq \operatorname{deg}\left(\Delta_{Y, \psi}\right)-1 \text {. }
$$

The desired inequality

$$
\bar{t}_{X}(\phi) \geq \operatorname{deg}\left(\Delta_{X, \phi}\right)-1
$$

is an immediate consequence of this fact and the observation that the Alexander polynomial depends only on the fundamental group of $X$.

3C. The Alexander norm. Let $X$ be a compact connected CW-complex. We write $H:=H_{1}(X ; \mathbb{Z}) /$ torsion and also $\Delta_{X}=\sum_{h \in H} a_{h} h$. Let

$$
\phi \in H^{1}(X ; \mathbb{Q})=\operatorname{Hom}\left(\pi_{1}(X), \mathbb{Q}\right)=\operatorname{Hom}(H, \mathbb{Q}) .
$$

Following [McMullen 2002], we define the Alexander norm of $\phi$ by

$$
a_{X}(\phi):=\max \left\{\phi(h)-\phi(g) \mid a_{g} \neq 0 \text { and } a_{h} \neq 0\right\} .
$$

It is straightforward to see that $a_{X}$ is indeed a norm on $H^{1}(X ; \mathbb{Q})$. As in the proof of Proposition 3.2, we use that fact that the Alexander polynomial and thus the Alexander norm depend only on the fundamental group of $X$. More precisely, if $f: Y \rightarrow X$ is a map of compact connected CW-complexes that induces an isomorphism of fundamental groups, then

$$
f_{*}\left(\Delta_{Y}\right)=\Delta_{X} \in \mathbb{Z}\left[H_{1}(X ; \mathbb{Z}) / \text { torsion }\right],
$$

and thus, for any $f \in H^{1}(X ; \mathbb{Q})=\operatorname{Hom}\left(\pi_{1}(X), \mathbb{Q}\right)$, we have

$$
a_{Y}\left(\phi \circ f^{*}\right)=a_{X}(\phi) .
$$

We begin with the following theorem due to McMullen [2002]. 
Theorem 3.3. Let $N$ be a 3-manifold with empty or toroidal boundary and with $b_{1}(N) \geq 2$. Then

$$
a_{N}(\phi) \leq x_{N}(\phi) \quad \text { for any } \phi \in H^{1}(N ; \mathbb{Q}) .
$$

Furthermore, equality holds for quasifibered classes.

Proof. Let $N$ be a 3-manifold with empty or toroidal boundary and with $b_{1}(N) \geq 2$. McMullen [2002, Theorem 1.1] showed that

$$
a_{N}(\phi) \leq x_{N}(\phi) \text { for any } \phi \in H^{1}(N ; \mathbb{Q})
$$

and that equality holds for all integral fibered classes. Since $a_{N}$ and $x_{N}$ are homogeneous, it follows immediately that equality also holds for all fibered classes and, in fact, for all quasifibered classes.

The following analogous theorem, which says that the Alexander norm also gives lower bounds on the Thurston norm and the Turaev complexity function, is due to Turaev [2002].

Theorem 3.4. Let $X$ be a finite 2-complex with $b_{1}(X) \geq 2$ and such that $\partial X=\varnothing$. Then

$$
a_{X}(\phi) \leq \bar{t}_{X}(\phi) \leq t_{X}(\phi) \quad \text { for any } \phi \in H^{1}(X ; \mathbb{Q}) .
$$

Proof. Let $Y$ be a finite 2-complex with $b_{1}(Y) \geq 2$ and such that $\partial Y=\varnothing$. Then by [Turaev 2002, Theorem 3.1], we have

$$
a_{Y}(\psi) \leq t_{Y}(\psi) \quad \text { for any } \psi \in H^{1}(Y ; \mathbb{Q}) .
$$

The theorem now follows immediately from combining this result with the definition of $\bar{t}_{X}(\phi)$ and (1).

\section{Proofs}

4A. The Thurston norm and the Turaev complexity function for closed 3-manifolds. The combination of Propositions 3.1, 3.2 and 2.1 gives us the following theorem showing that the Thurston norm of a closed 3-manifold need not agree with Turaev complexity function of its 2-skeleton.

Theorem 4.1. Let $N \neq S^{1} \times S^{2}$ be a closed 3-manifold and let $\phi \in H^{1}(N ; \mathbb{Z})$ be a primitive fibered class. Then

$$
\bar{t}_{N}(\phi)=x_{N}(\phi)+1 .
$$

We also prove:

Proposition 4.2. There exists a finite 2-complex $X$ with $\partial X=\varnothing$ such that $\bar{t}_{X}$ does not satisfy the triangle inequality, i.e., $\bar{t}_{X}$ is not a norm. 
Proof. Let $N$ be a fibered 3-manifold with $b_{1}(N)=2$. We write $X=N^{(2)}$ for some triangulation of $N$. As we mentioned in Section 2A, by [Thurston 1986] there exists an open 2-dimensional cone $C \subset H^{1}(N ; \mathbb{Q})$ such that all classes in $C$ are fibered and such that $x_{N}$ is a linear function on $C$.

Given $\phi \in H^{1}(N ; \mathbb{Z})$ we denote by

$$
\operatorname{div}(\phi):=\max \left\{k \in \mathbb{N} \mid \text { there exists } \psi \in H^{1}(N ; \mathbb{Z}) \text { with } \phi=k \psi\right\}
$$

the divisibility of $\phi$. It follows from Theorem 4.1 and the homogeneity of the Thurston norm and the Turaev complexity function that

$$
\bar{t}_{X}(\phi)=x_{N}(\phi)+\operatorname{div}(\phi) \quad \text { for any } \phi \in H^{1}(N ; \mathbb{Z}) \cap C .
$$

We prove the following claim.

Claim. There exist $\alpha, \beta \in C$ with $\operatorname{div}(\alpha)+\operatorname{div}(\beta)<\operatorname{div}(\alpha+\beta)$.

Pick two primitive vectors $\phi, \psi \in C$ which are not collinear. Since $\phi$ and $\psi$ lie in the cone $C$, it follows that any nonnegative linear combination of $\phi$ and $\psi$ also lies in $C$.

Select a coordinate system for $H^{1}(N ; \mathbb{Z})$, that is, choose an identification of $H^{1}(N ; \mathbb{Z})$ with $\mathbb{Z}^{2}$. Since $\phi$ is primitive, we can assume that $\phi=(1,0)$. Since $\psi$ is also primitive, we know that $\psi=(x, y)$ for some coprime $x$ and $y$. Since $\phi$ and $\psi$ are not collinear, $y \neq 0$. Choose a prime $p>1+|y|$. We consider $\alpha=(1,0)$ and $\beta=(p x+(p-1), p y)$. Note that $p$ can not divide $p x+p-1=p(x+1)-1$. It follows that $\operatorname{div}(\beta)=\operatorname{gcd}(p x+(p-1), p y) \leq|y|$. Evidently $\operatorname{div}(\alpha)=1$. Now

$\operatorname{div}(\alpha+\beta)=\operatorname{div}(p x+p, p y)=\operatorname{gcd}(p x+p, p y) \geq p>1+|y| \geq \operatorname{div}(\alpha)+\operatorname{div}(\beta)$.

This concludes the proof of the claim.

If we combine the claim and the linearity of $x_{N}$ on $C$ with equality (2), then we obtain that

$$
\begin{aligned}
\bar{t}_{X}(\alpha+\beta)=x_{N}(\alpha+\beta)+\operatorname{div}(\alpha+\beta) & =x_{N}(\alpha)+x_{N}(\beta)+\operatorname{div}(\alpha+\beta) \\
& >x_{N}(\alpha)+\operatorname{div}(\alpha)+x_{N}(\beta)+\operatorname{div}(\beta) \\
& =\bar{t}_{X}(\alpha)+\bar{t}_{X}(\beta) .
\end{aligned}
$$

We have shown that $\bar{t}_{X}$ does not satisfy the triangle inequality.

4B. The Alexander norm of finite covers of 3-manifolds. We begin with the following theorem. We state it in slightly greater generality than we actually need, since the result has independent interest.

Theorem 4.3. Let $N \neq S^{1} \times D^{2}$ be an aspherical 3-manifold with empty or toroidal boundary. If $N$ is neither a Nil-manifold nor a Sol-manifold, there exists a finite 
cover $p: \tilde{N} \rightarrow N$ such that $b_{1}(\tilde{N}) \geq 2$ and such that

$$
a_{\tilde{N}}\left(p^{*} \phi\right)=x_{\tilde{N}}\left(p^{*} \phi\right) \text { for any } \phi \in H^{1}(N ; \mathbb{Q}) .
$$

The proof of the theorem will require the remainder of Section 4B. The theorem was proved for graph manifolds by Nagel [2014]. We will therefore restrict ourselves to the case of manifolds that are not (closed) graph manifolds. The main ingredient in our proof of Theorem 4.3 will be the following theorem, a consequence of the seminal work of Agol [2008; 2013], Wise [2009; 2012b; 2012a], Przytycki and Wise [2014; 2012] and Liu [2013]. We summarize the main points of the proof for the convenience of the reader.

Theorem 4.4. Let $N$ be an irreducible 3-manifold with empty or toroidal boundary that is not a closed graph manifold. Then there exists a finite cover $p: \tilde{N} \rightarrow N$ such that, for any $\phi \in H^{1}(N ; \mathbb{Q})$, the pull-back $p^{*} \phi$ is quasifibered.

Proof. Let $N$ be an irreducible 3-manifold that is not a closed graph manifold. It follows from [Agol 2013; Wise 2009; 2012b; 2012a; Przytycki and Wise 2014; 2012; Liu 2013] that $\pi_{1}(N)$ is virtually RFRS, i.e., $\pi_{1}(N)$ admits a finite index subgroup which is RFRS (residually finite rationally solvable). The precise definition of RFRS, references for which can be found in [Aschenbrenner et al. 2015], is not of concern to us. What matters is that Agol [2008, Theorem 5.1] (see also [Friedl and Kitayama 2014, Theorem 5.1]) showed that if $\psi$ lies in $H^{1}(N ; \mathbb{Q})$ and if $N$ is an irreducible 3-manifold such that $\pi_{1}(N)$ is virtually RFRS, then there exists a finite cover $p: \hat{N} \rightarrow N$ such that $p^{*} \psi$ lies in the closure of a fibered cone of $\hat{N}$.

By picking one class in each cone of the Thurston norm ball of $N$ and iteratively applying Agol's theorem, one can easily show that there exists a finite cover $p: \tilde{N} \rightarrow N$ such that for any $\phi \in H^{1}(N ; \mathbb{Q})$ the pull-back $p^{*} \phi$ lies in the closure of a fibered cone of $\tilde{N}$. We refer to [Friedl and Vidussi 2015, Corollary 5.2] for details.

If $N$ is a graph manifold with nonempty boundary, then the conclusion of Theorem 4.4 also follows from facts that are more classical. This argument is not used anywhere else in the paper, but since it is perhaps of independent interest we give a very quick sketch of the argument.

Proof of Theorem 4.4 if $N$ is a graph manifold. Let $N$ be a graph manifold with boundary. It follows from [Wang and Yu 1997, Theorem 0.1] and classical arguments (see e.g., [Aschenbrenner and Friedl 2013, Section 4.3.4.3] and [Hempel 1987]) that there exists a finite cover $\tilde{N}$ of $N$ that is fibered and such that if $\left\{N_{v}\right\}_{v \in V}$ denotes the set of JSJ components of $\tilde{N}$, then each $N_{v}$ is of the form $S^{1} \times \Sigma_{v}$ for some surface $\Sigma_{v}$. (For the meaning of JSJ components, see [Aschenbrenner et al. 2015, Section 1.6].) 
For each $v \in V$ we write $t_{v}=S^{1} \times P_{v}$, where $P_{v} \in \Sigma_{v}$ is a point. It follows from [Eisenbud and Neumann 1985, Theorem 4.2] that a class $\phi \in H^{1}(\tilde{N} ; \mathbb{Q})$ is fibered if and only if $\phi\left(t_{v}\right) \neq 0$ for all $v \in V$. Since $\tilde{N}$ is fibered it now follows that all classes in $H^{1}(\tilde{N} ; \mathbb{Q})$ outside of finitely many hyperplanes are fibered. Hence all classes in $H^{1}(\tilde{N} ; \mathbb{Q})$ are quasifibered.

We can now move on to the proof of Theorem 4.3. Note that arguments similar to the proof of Theorem 4.3 were also used in [Friedl and Vidussi 2014; 2015].

Proof of Theorem 4.3. Let $N \neq S^{1} \times D^{2}$ be an irreducible 3-manifold with empty or toroidal boundary that is not a closed graph manifold. Since we assumed that $N \neq S^{1} \times D^{2}$, it now follows from Agol's theorem [2013] and classical 3-manifold topology that $N$ has a finite cover with $b_{1}$ at least two. (We refer to [Aschenbrenner et al. 2015] for details.) We can therefore assume that we already have $b_{1}(N) \geq 2$.

By Theorem 4.4 there exists a finite cover $p: \tilde{N} \rightarrow N$ such that for any $\phi$ in $H^{1}(N ; \mathbb{Q})$, the pull-back $p^{*} \phi$ is quasifibered. Note that Betti numbers never decrease by going to finite covers, i.e., we have $b_{1}(\tilde{N}) \geq b_{1}(N) \geq 2$. It follows from Theorem 3.3 that

$$
a_{\tilde{N}}\left(p^{*} \phi\right)=x_{\tilde{N}}\left(p^{*} \phi\right) \quad \text { for any } \phi \in H^{1}(N ; \mathbb{Q}) .
$$

This concludes the proof of the theorem.

4C. Proof of Theorem 1.2. Before we turn to the proof of Theorem 1.2 we prove the following well-known lemma.

Lemma 4.5. If $N$ is the exterior of a link in a rational homology sphere, then any class $\phi \in H^{1}(N ; \mathbb{Z})$ is dual to a surface $\Sigma$ of minimal complexity such that all components of $\Sigma$ have nonempty boundary.

Proof. Let $N$ be the exterior of a link in a rational homology sphere. It follows from a Mayer-Vietoris argument that the map $H_{1}(\partial N ; \mathbb{Q}) \rightarrow H_{1}(N ; \mathbb{Q})$ is surjective. It follows from Poincaré duality and the Universal Coefficient Theorem that the boundary map $\partial: H_{2}(N, \partial N ; \mathbb{Z}) \rightarrow H_{1}(\partial N ; \mathbb{Z})$ has finite kernel. Since $H_{2}(N, \partial N ; \mathbb{Z}) \cong H^{1}(N ; \mathbb{Z}) \cong \operatorname{Hom}\left(H_{1}(N ; \mathbb{Z}), \mathbb{Z}\right)$ is torsion-free it follows that the boundary map $\partial: H_{2}(N, \partial N ; \mathbb{Z}) \rightarrow H_{1}(\partial N ; \mathbb{Z})$ is in fact injective. In particular this implies that closed surfaces represent the trivial homology class in $(N, \partial N)$. Now let $\phi \in H^{1}(N ; \mathbb{Z})$, and let $\Sigma$ be a properly embedded minimal-complexity surface dual to $\phi$. By the above observation, the closed components of $\Sigma$ are null-homologous. It follows that the union of the components of $\Sigma$ with nontrivial boundary represents the same homology as $\Sigma$. Since removing components can never increase the complexity, we have shown that $\phi$ is dual to a surface $\Sigma$ of minimal complexity such that all components of $\Sigma$ have nonempty boundary. 
In the previous sections we collected all the tools that now allow us to finally complete the proof of Theorem 1.2.

Theorem 1.2. Let $N$ be the exterior of a link in a rational homology sphere. If $N$ is irreducible, then for any $\phi \in H^{1}(N ; \mathbb{Q})$ we have

$$
\bar{t}_{N}(\phi)=x_{N}(\phi) \text {. }
$$

Proof. It remains to prove that $\bar{t}_{N}(\phi) \geq x_{N}(\phi)$. Let $N$ be the exterior of a link in a rational homology sphere. Suppose that $N$ is irreducible. Let $\phi \in H^{1}(N ; \mathbb{Q})$. It suffices to show that if $Y$ is a finite 2-complex $Y$ with $\partial Y=\varnothing$ and if $f: \pi_{1}(Y) \rightarrow \pi_{1}(N)$ is an isomorphism, then

$$
t_{Y}(\phi \circ f) \geq x_{N}(\phi) .
$$

So let $Y$ and $f$ be as above. By a slight abuse of notation we denote $\phi \circ f: \pi_{1}(Y) \rightarrow \mathbb{Q}$ by $\phi$ as well.

By Theorem 4.3 there exists a finite cover $p: \tilde{N} \rightarrow N$ such that $b_{1}(\tilde{N}) \geq 2$ and such that

$$
a_{\tilde{N}}\left(p^{*} \phi\right)=x_{\tilde{N}}\left(p^{*} \phi\right) .
$$

We write $\pi=\pi_{1}(N)$ and $\tilde{\pi}:=\pi_{1}(\tilde{N})$, and we denote by $p: \tilde{Y} \rightarrow Y$ the finite cover corresponding to $f^{-1}(\tilde{\pi})$. Note that $\tilde{Y}$ is also a finite 2-complex with $\partial \tilde{Y}=\varnothing$. It follows immediately from the definitions that

$$
x_{\tilde{N}}\left(p^{*} \phi\right) \leq[\pi: \tilde{\pi}] \cdot x_{N}(\phi) \text { and } t_{\tilde{Y}}\left(p^{*} \phi\right) \leq[\pi: \tilde{\pi}] \cdot t_{Y}(\phi) .
$$

In fact, Gabai [1983, Corollary 6.13] showed that the above is an equality for the Thurston norm, i.e., we have the equality:

$$
x_{\tilde{N}}\left(p^{*} \phi\right)=[\pi: \tilde{\pi}] \cdot x_{N}(\phi) .
$$

Combining the above results with Theorem 3.4, we see that

$$
[\pi: \tilde{\pi}] \cdot t_{Y}(\phi) \geq t_{\tilde{Y}}\left(p^{*} \phi\right) \geq a_{\tilde{N}}\left(p^{*} \phi\right)=x_{\tilde{N}}\left(p^{*} \phi\right)=[\pi: \tilde{\pi}] \cdot x_{N}(\phi) .
$$

This concludes the proof the theorem.

4D. Fundamental group complexity. Let $X$ be a finite 2-complex with $\partial X=\varnothing$, and $\phi \in H^{1}(X ; \mathbb{Z})=\operatorname{Hom}\left(\pi_{1}(X), \mathbb{Z}\right)$. Turaev [2002] describes a method by which we can compute $t_{X}(\phi)$ using cocycles. We start by orienting edges (i.e., open 1 -cells) of $X$, and then select a $\mathbb{Z}$-valued cellular cocycle $k$ on $X$ representing $\phi$. We let

$$
|k|=\sum_{e}\left(n_{e} / 2-1\right)|k(e)|,
$$

where $e$ ranges over all edges in $X, k(e) \in \mathbb{Z}$ is the value of $k$ on $e$, and $n_{e}$ is the 
number of 2-cells adjacent to $e$, counted with multiplicity. (Note that $n_{e} \geq 2$ since $\partial X=\varnothing$.) Turaev [2002, Section 1.6] proves that $t_{X}(\phi)$ is the minimum value of $|k|$ as $k$ ranges over all cellular cocycles representing $\phi$.

When the 0 -skeleton of $X$ consists of a single vertex, the 2-complex determines a group presentation $P$ for $\pi_{1}(X)$, and hence $|k|$ can be defined on the level of presentations.

Given a finite presentation $P=\left\langle x_{1}, \ldots, x_{m} \mid r_{1}, \ldots, r_{n}\right\rangle$, following [Turaev 2002], we denote by $\#\left(x_{i}\right)$ the number of appearances of $x_{i}^{ \pm 1}$ in the words $r_{1}, \ldots, r_{n}$. We say that $P$ is a good presentation if each $\#\left(x_{i}\right) \geq 2$. We are interested in good presentations, since it is straightforward to see that the canonical 2-complex corresponding to a good presentation has empty boundary. Also note that any finitely presented group admits a good presentation. Indeed, if $\#\left(x_{i}\right)=1$, then we can eliminate $x_{i}$ using a Tietze move. If $\#\left(x_{i}\right)=0$, then we can add a trivial relator $x_{i} x_{i}^{-1}$.

Now let $P=\left\langle x_{1}, \ldots, x_{m} \mid r_{1}, \ldots, r_{n}\right\rangle$ be a good presentation for a group $\pi$, and let $\phi$ be a homomorphism $\phi: \pi \rightarrow \mathbb{Z}$. We define

$$
t_{P}(\phi)=\sum_{i}\left(\#\left(x_{i}\right) / 2-1\right)\left|\phi\left(x_{i}\right)\right| .
$$

Furthermore we define $\bar{t}_{\pi}(\phi)$ to be the minimum of $t_{P}(\phi)$ as $P$ ranges over all good presentations of $\pi$. We extend the definition in the usual way for rational cohomology classes $\phi \in H^{1}(X ; \mathbb{Q})$.

Lemma 4.6. Let $X$ be a finite 2-complex with $\partial X=\varnothing$ and $\phi \in H^{1}(X ; \mathbb{Q})$. We write $\pi=\pi_{1}(X)$. Then

$$
\bar{t}_{X}(\phi) \leq \bar{t}_{\pi}(\phi) .
$$

Proof. Given a good presentation $P$ for $\pi$, we construct the canonical finite 2complex $Y$ with $\pi_{1}(Y) \cong \pi$. Let $k$ be the unique 1-cocycle representing $\phi$. A straightforward argument shows that $\bar{t}_{X}(\phi) \leq|k|=t_{P}(\phi)$; see also [Turaev 2002, Section 1.8]. Since this is true for any good presentation of $\pi_{1}(X)$, we have $\bar{t}_{X}(\phi) \leq \bar{t}_{\pi}(\phi)$.

Example 4.7. Let $\pi$ the fundamental group of the exterior of a knot $K$ in the 3 -sphere. Let $\phi$ be the abelianization homomorphism, mapping a meridian to 1 . If $P$ is a Wirtinger presentation corresponding to a diagram for $K$, then one sees easily that $t_{P}(\phi)$ is the number of crossings of the diagram.

It is usually possible to find presentations yielding a smaller value $t_{P}(\phi)$. Let $\Sigma$ be a Seifert surface for $K$ having minimal genus $g$. By splitting $\pi$ along $\pi_{1}(\Sigma)$, we obtain an $\mathrm{HNN}$-decomposition for $\pi$ of the form

$$
\left.\langle A, x| \mu(b)=x b x^{-1} \text { for all } b \in \pi_{1}(\Sigma)\right\rangle,
$$


where $A$ is the fundamental group of the knot exterior split along $\Sigma$, and

$$
\mu: \pi_{1}(\Sigma) \rightarrow A
$$

is injective. For such a presentation $P$, we have $t_{P}(\phi)=2 g-1$. It follows by the next result that this value is the smallest possible; i.e., $\bar{t}_{\pi}(\phi)=2 g-1$.

Theorem 4.8. Let $N$ be the exterior of a link in a rational homology sphere with group $\pi$. If $N$ is irreducible, then for any $\phi \in H^{1}(N ; \mathbb{Q})$ such that $\Delta_{N, \phi} \neq 0$, we have

$$
\bar{t}_{N}(\phi)=\bar{t}_{\pi}(\phi)=x_{N}(\phi) .
$$

Remark. Turaev [2002] gives several examples of knot groups and presentations of minimal complexity. He states that it would be interesting to find other examples. Theorem 4.8 shows how to construct presentations of minimal complexity for any knot in a rational homology sphere.

Proof. By Lemma 4.6 and Theorem 1.2, it suffices to prove that $\bar{t}_{\pi}(\phi) \leq x_{N}(\phi)$, for any $\phi \in H^{1}(N ; \mathbb{Q})$. By the homogeneity of the Turaev function and the Thurston norm we may assume that $\phi$ is an integral primitive cohomology class.

Consider a Thurston norm-minimizing surface $\Sigma \subset N$ for $\phi$. Our assumption that $\Delta_{N, \phi}$ is not identically zero ensures that the first Betti number of $\operatorname{Ker}(\phi)$ is finite. By a short argument in the beginning of the proof of McMullen [2002, Proposition 6.1], the surface $\Sigma$ is connected. Its boundary is nonempty by Lemma 4.5. Splitting $\pi$ along $\pi_{1}(\Sigma)$, as above, we obtain a presentation $P$ with complexity $2 g-1$, where $g$ is the genus of $\Sigma$. Since $t_{N}(\phi)=2 g-1$, we are done.

We conclude this section with the following conjecture:

Conjecture 4.9. Let $X$ be a finite 2-complex with $\partial X=\varnothing$. Then

$$
\bar{t}_{X}(\phi)=\bar{t}_{\pi_{1}(X)}(\phi) \quad \text { for any } \phi \in H^{1}(X ; \mathbb{Q}) .
$$

Note that an affirmative answer to this question together with Theorems 1.1 and 1.2 would show that the conclusion of Theorem 4.8 holds for any irreducible link complement $N$, without any assumptions on $\phi$.

\section{Disconnected minimal dual graphs}

It is natural to ask whether one can always realize the Turaev norm of a primitive cohomology class by a connected graph. In this final section of the paper we will see that this is not the case. More precisely, we have the following theorem.

Theorem 5.1. Given any $n$ there exists a 2-complex $X$ with $\partial X=\varnothing$ and a primitive class $\phi \in H^{1}(\pi ; \mathbb{Z})$ such that for any 2 -complex $Y$ with $\pi_{1}(Y)=\pi_{1}(X)$ and with $\partial Y=\varnothing$ the following holds: any graph $\Gamma$ in $Y$ that represents $\phi$ with $\bar{t}_{X}(\phi)=\chi_{-}(\Gamma)$ has at least $n$ components. 
Proof. We consider the good presentation

$$
P=\left\langle a_{1}, \ldots, a_{n}, x_{1}, \ldots, x_{n} \mid\left[x_{i}, a_{i}\right], i=1, \ldots, n\right\rangle,
$$

and we denote by $X$ the corresponding 2-complex, which is just the join of $n$ tori $T_{1}, \ldots, T_{n}$. Clearly $\partial X=\varnothing$.

We write $\pi=\pi_{1}(X)$. The group $\pi$ is the free product of $n$ free abelian groups $\left\langle a_{i}, x_{i} \mid\left[a_{i}, x_{i}\right]\right\rangle, i=1, \ldots, n$ of rank two. We consider the epimorphism $\phi: \pi \rightarrow \mathbb{Z}$ that is defined by $\phi\left(a_{i}\right)=0, i=1, \ldots, n$ and $\phi\left(x_{i}\right)=1, i=1, \ldots, n$. It is clear that on each torus $T_{i}$ there exists a circle, disjoint from the gluing point, such that the union of these circles is dual to $\phi$. We thus see that $\bar{t}_{X}(\phi)=0$.

Now let $Y$ be a 2-complex with $\pi_{1}(Y)=\pi$ and with $\partial Y=\varnothing$. Let $\Gamma$ be a graph on $Y$ which is dual to $\phi$ with $\chi_{-}(\Gamma)=0$. We will show that $\Gamma$ has at least $n$ components. Note that $\chi_{-}(\Gamma)=0$ implies that any component of $\Gamma$ is either a point or a circle. We denote by $m$ the number of components of $\Gamma$ that are circles. We will see that $m \geq n$.

Claim. The module $H_{1}\left(Y ; \mathbb{Q}\left[t^{ \pm 1}\right]\right)$ is isomorphic to

$$
\mathbb{Q}\left[t^{ \pm 1}\right]^{n-1} \oplus \bigoplus_{i=1}^{n} \mathbb{Q}\left[t^{ \pm 1}\right] /(t-1) .
$$

We first note that $H_{1}\left(Y ; \mathbb{Q}\left[t^{ \pm 1}\right]\right)=H_{1}\left(X ; \mathbb{Q}\left[t^{ \pm 1}\right]\right)$. A straightforward application of Fox calculus (see [Fox 1953]) shows that

$$
H_{1}\left(X ; \mathbb{Q}\left[t^{ \pm 1}\right]\right) \cong \mathbb{Q}\left[t^{ \pm 1}\right]^{n-1} \oplus \bigoplus_{i=1}^{n} \mathbb{Q}\left[t^{ \pm 1}\right] /(t-1) .
$$

This concludes the proof of the claim.

Now we write $W=Y \backslash \Gamma \times(-1,1)$. The usual Meyer-Vietoris sequence with $\mathbb{Q}\left[t^{ \pm 1}\right]$-coefficients corresponding to $Y=W \cup \Gamma \times[-1,1]$ gives rise to the exact sequence

$$
\cdots \rightarrow H_{1}\left(\Gamma ; \mathbb{Q}\left[t^{ \pm 1}\right]\right) \stackrel{\iota_{-}-t \iota_{+}}{\longrightarrow} H_{1}\left(W ; \mathbb{Q}\left[t^{ \pm 1}\right]\right) \rightarrow
$$

$$
H_{1}\left(Y ; \mathbb{Q}\left[t^{ \pm 1}\right]\right) \rightarrow H_{0}\left(\Gamma ; \mathbb{Q}\left[t^{ \pm 1}\right]\right) \rightarrow \cdots
$$

Note that $\phi$ vanishes on $\Gamma$ and $W$. It follows that $H_{*}\left(\Gamma ; \mathbb{Q}\left[t^{ \pm 1}\right]\right)$ and $H_{*}\left(W ; \mathbb{Q}\left[t^{ \pm 1}\right]\right)$ are free $\mathbb{Q}\left[t^{ \pm 1}\right]$-modules. Furthermore, by the above discussion of $\Gamma$ we know that $H_{1}\left(\Gamma ; \mathbb{Q}\left[t^{ \pm 1}\right]\right) \cong \mathbb{Q}\left[t^{ \pm 1}\right]^{m}$. It follows immediately from the above exact sequence and the classification of modules over PIDs that the torsion submodule of $H_{1}\left(Y ; \mathbb{Q}\left[t^{ \pm 1}\right]\right)$ is generated by $m$ elements.

On the other hand, we had just seen that the torsion submodule of $H_{1}\left(Y ; \mathbb{Q}\left[t^{ \pm 1}\right]\right)$ is isomorphic to $\oplus_{i=1}^{n} \mathbb{Q}\left[t^{ \pm 1}\right] /(t-1)$. It follows from the classification of modules over the PID $\mathbb{Q}\left[t^{ \pm 1}\right]$ that the minimal number of generators of the torsion submodule of $H_{1}\left(Y ; \mathbb{Q}\left[t^{ \pm 1}\right]\right)$ is $n$. Putting everything together we deduce that $m \geq n$. 


\section{Acknowledgments}

Friedl gratefully acknowledges the support provided by the SFB 1085 "Higher Invariants" at the Universität Regensburg, funded by the Deutsche Forschungsgemeinschaft (DFG). Silver and Williams thank the Simons Foundation for its support. We wish to thank the referee for carefully reading our paper and for pointing out a well-hidden mistake in an argument.

\section{References}

[Agol 2008] I. Agol, “Criteria for virtual fibering”, J. Topol. 1:2 (2008), 269-284. MR Zbl

[Agol 2013] I. Agol, "The virtual Haken conjecture", Doc. Math. 18 (2013), 1045-1087. MR Zbl

[Aschenbrenner and Friedl 2013] M. Aschenbrenner and S. Friedl, 3-manifold groups are virtually residually $p$, Memoirs of the American Mathematical Society 1058, 2013. MR

[Aschenbrenner et al. 2015] M. Aschenbrenner, S. Friedl, and H. Wilton, 3-manifold groups, European Mathematical Society (EMS), Zürich, 2015. MR Zbl

[Eisenbud and Neumann 1985] D. Eisenbud and W. Neumann, Three-dimensional link theory and invariants of plane curve singularities, Annals of Mathematics Studies 110, Princeton University Press, 1985. MR Zbl

[Fox 1953] R. H. Fox, "Free differential calculus, I: Derivation in the free group ring", Ann. of Math.

(2) 57 (1953), 547-560. MR Zbl

[Fox 1954] R. H. Fox, "Free differential calculus, II: The isomorphism problem of groups", Ann. of Math. (2) 59 (1954), 196-210. MR Zbl

[Friedl and Kim 2006] S. Friedl and T. Kim, "The Thurston norm, fibered manifolds and twisted Alexander polynomials", Topology 45:6 (2006), 929-953. MR Zbl

[Friedl and Kitayama 2014] S. Friedl and T. Kitayama, "The virtual fibering theorem for 3-manifolds", Enseign. Math. 60:1-2 (2014), 79-107. MR Zbl

[Friedl and Vidussi 2014] S. Friedl and S. Vidussi, "Minimal genus in 4-manifolds with a free circle action", Adv. Math. 250 (2014), 570-587. MR Zbl

[Friedl and Vidussi 2015] S. Friedl and S. Vidussi, "The Thurston norm and twisted Alexander polynomials", J. Reine Angew. Math. 707 (2015), 87-102. MR Zbl

[Friedl et al. 2015] S. Friedl, D. S. Silver, and S. G. Williams, "Splittings of knot groups", Math. Ann. 362:1-2 (2015), 401-424. MR Zbl

[Gabai 1983] D. Gabai, "Foliations and the topology of 3-manifolds", J. Differential Geom. 18:3 (1983), 445-503. MR Zbl

[Gabai 1987] D. Gabai, "Foliations and the topology of 3-manifolds, III", J. Differential Geom. 26:3 (1987), 479-536. MR Zbl

[Hempel 1987] J. Hempel, "Residual finiteness for 3-manifolds", pp. 379-396 in Combinatorial group theory and topology (Alta, Utah, 1984), edited by S. M. Gersten and J. R. Stallings, Ann. of Math. Stud. 111, Princeton University Press, 1987. MR Zbl

[Hillman 2012] J. Hillman, Algebraic invariants of links, 2nd ed., Series on Knots and Everything 52, World Scientific, Hackensack, NJ, 2012. MR Zbl

[Kronheimer 1999] P. B. Kronheimer, "Minimal genus in $S^{1} \times M^{3}$ ", Invent. Math. 135:1 (1999), 45-61. MR Zbl

[Liu 2013] Y. Liu, "Virtual cubulation of nonpositively curved graph manifolds", J. Topol. 6:4 (2013), 793-822. MR Zbl 
[McMullen 2002] C. T. McMullen, "The Alexander polynomial of a 3-manifold and the Thurston norm on cohomology", Ann. Sci. École Norm. Sup. (4) 35:2 (2002), 153-171. MR Zbl

[Nagel 2014] M. Nagel, "Minimal genus in circle bundles over 3-manifolds", preprint, 2014. arXiv [Przytycki and Wise 2012] P. Przytycki and D. T. Wise, "Mixed 3-manifolds are virtually special", preprint, 2012. arXiv

[Przytycki and Wise 2014] P. Przytycki and D. T. Wise, "Graph manifolds with boundary are virtually special”, J. Topol. 7:2 (2014), 419-435. MR Zbl

[Thurston 1986] W. P. Thurston, "A norm for the homology of 3-manifolds", pp. 99-130 Memoirs of the American Mathematical Society 339, 1986. MR

[Tischler 1970] D. Tischler, "On fibering certain foliated manifolds over $S^{1}$ ”, Topology 9 (1970), 153-154. MR Zbl

[Turaev 2001] V. Turaev, Introduction to combinatorial torsions, Birkhäuser, Basel, 2001. MR Zbl

[Turaev 2002] V. Turaev, "A norm for the cohomology of 2-complexes", Algebr. Geom. Topol. 2 (2002), 137-155. MR Zbl

[Wang and Yu 1997] S. Wang and F. Yu, "Graph manifolds with non-empty boundary are covered by surface bundles”, Math. Proc. Cambridge Philos. Soc. 122:3 (1997), 447-455. MR Zbl

[Wise 2009] D. T. Wise, "Research announcement: the structure of groups with a quasiconvex hierarchy", Electron. Res. Announc. Math. Sci. 16 (2009), 44-55. MR Zbl

[Wise 2012a] D. T. Wise, From riches to raags: 3-manifolds, right-angled Artin groups, and cubical geometry, CBMS Regional Conference Series in Mathematics 117, American Mathematical Society, 2012. MR Zbl

[Wise 2012b] D. T. Wise, “The structure of groups with a quasi-convex hierarchy", preprint, 2012, available at https://goo.gl/ucBthh.

Received July 30, 2015. Revised February 23, 2016.

\section{STEFAN FRIEDL}

FAKULTÄT FÜR MATHEMATIK

UNIVERSITÄT REGENSBURG

D-93047 REGENSBURG

GERMANY

sfriedl@gmail.com

\section{DANIEL S. SILVER}

DEPARTMENT OF MATHEMATICS AND Statistics

University of SOUTh Alabama

MOBILE, AL 36688-0002

UNITED STATES

silver@southalabama.edu

SUSAN G. WILLIAMS

DEPARTMENT OF MATHEMATICS AND STATistics

University of SOUTH Alabama

MoBILE, AL 36688-0002

UNITED STATES

swilliam@southalabama.edu 


\title{
PACIFIC JOURNAL OF MATHEMATICS
}

Founded in 1951 by E. F. Beckenbach (1906-1982) and F. Wolf (1904-1989)

$$
\text { msp.org/pjm }
$$

\section{EDITORS}

\author{
Don Blasius (Managing Editor) \\ Department of Mathematics \\ University of California \\ Los Angeles, CA 90095-1555 \\ blasius@math.ucla.edu
}

\author{
Paul Balmer \\ Department of Mathematics \\ University of California \\ Los Angeles, CA 90095-1555 \\ balmer@math.ucla.edu \\ Robert Finn \\ Department of Mathematics \\ Stanford University \\ Stanford, CA 94305-2125 \\ finn@math.stanford.edu \\ Sorin Popa \\ Department of Mathematics \\ University of California \\ Los Angeles, CA 90095-1555 \\ popa@math.ucla.edu
}

\author{
Vyjayanthi Chari \\ Department of Mathematics \\ University of California \\ Riverside, CA 92521-0135 \\ chari@math.ucr.edu \\ Kefeng Liu \\ Department of Mathematics \\ University of California \\ Los Angeles, CA 90095-1555 \\ liu@math.ucla.edu \\ Igor Pak \\ Department of Mathematics \\ University of California \\ Los Angeles, CA 90095-1555 \\ pak.pjm@gmail.com \\ Paul Yang \\ Department of Mathematics \\ Princeton University \\ Princeton NJ 08544-1000 \\ yang@math.princeton.edu
}

\section{PRODUCTION}

Silvio Levy, Scientific Editor, production@msp.org

\section{SUPPORTING INSTITUTIONS}

ACADEMIA SINICA, TAIPEI

CALIFORNIA INST. OF TECHNOLOGY

STANFORD UNIVERSITY

UNIV. OF BRITISH COLUMBIA

UNIV. OF CALIFORNIA, BERKELEY

UNIV. OF CALIFORNIA, DAVIS

UNIV. OF CALIFORNIA, LOS ANGELES

UNIV. OF CALIFORNIA, RIVERSIDE

UNIV. OF CALIFORNIA, SAN DIEGO

UNIV. OF CALIF., SANTA BARBARA
KEIO UNIVERSITY

MATH. SCIENCES RESEARCH INSTITUTE

NEW MEXICO STATE UNIV.

OREGON STATE UNIV.
Daryl Cooper

Department of Mathematics

University of California

Santa Barbara, CA 93106-3080 cooper@math.ucsb.edu

Jiang-Hua Lu

Department of Mathematics

The University of Hong Kong

Pokfulam Rd., Hong Kong

jhlu@maths.hku.hk

$$
\text { Jie Qing }
$$

Department of Mathematics

University of California

Santa Cruz, CA 95064

qing@ cats.ucsc.edu

\author{
UNIV. OF CALIF., SANTA CRUZ \\ UNIV. OF MONTANA \\ UNIV. OF OREGON \\ UNIV. OF SOUTHERN CALIFORNIA \\ UNIV. OF UTAH \\ UNIV. OF WASHINGTON \\ WASHINGTON STATE UNIVERSITY
}

These supporting institutions contribute to the cost of publication of this Journal, but they are not owners or publishers and have no responsibility for its contents or policies.

See inside back cover or msp.org/pjm for submission instructions.

The subscription price for 2016 is US $\$ 440 /$ year for the electronic version, and \$600/year for print and electronic.

Subscriptions, requests for back issues and changes of subscriber address should be sent to Pacific Journal of Mathematics, P.O. Box 4163, Berkeley, CA 94704-0163, U.S.A. The Pacific Journal of Mathematics is indexed by Mathematical Reviews, Zentralblatt MATH, PASCAL CNRS Index, Referativnyi Zhurnal, Current Mathematical Publications and Web of Knowledge (Science Citation Index).

The Pacific Journal of Mathematics (ISSN 0030-8730) at the University of California, c/o Department of Mathematics, 798 Evans Hall \#3840, Berkeley, CA 94720-3840, is published twelve times a year. Periodical rate postage paid at Berkeley, CA 94704, and additional mailing offices. POSTMASTER: send address changes to Pacific Journal of Mathematics, P.O. Box 4163, Berkeley, CA 94704-0163.

PJM peer review and production are managed by EditFLOW ${ }^{\circledR}$ from Mathematical Sciences Publishers.

PUBLISHED BY

\section{I. mathematical sciences publishers}

nonprofit scientific publishing

http://msp.org/

(C) 2016 Mathematical Sciences Publishers 


\section{PACIFIC JOURNAL OF MATHEMATICS}

Volume $284 \quad$ No. $2 \quad$ October 2016

Spherical CR Dehn surgeries

Miguel ACOsta

Degenerate flag varieties and Schubert varieties: a characteristic free approach

283

Giovanni Cerulli Irelli, Martina LANini and Peter

LITTELMANN

Solitons for the inverse mean curvature flow

Gregory Drugan, HoJoo LEE and GLEN WHEELER

Bergman theory of certain generalized Hartogs triangles

\section{LUKE D. EDHOLM}

Transference of certain maximal Hilbert transforms on the torus

DASHAN FAN, HUOXIONG WU and FAYOU ZHAO

The Turaev and Thurston norms

STEFAN FriedL, DANIEL S. Silver and Susan G. WiLLiams

A note on nonunital absorbing extensions

JAMES GABE

On nonradial singular solutions of supercritical biharmonic equations

Zongming GuO, JUNCHENG WeI and Wen YANG

Natural commuting of vanishing cycles and the Verdier dual

\section{DAVID B. MASSEY}

The nef cones of and minimal-degree curves in the Hilbert schemes of points on certain surfaces

ZHENBo QIN and YUPING TU

Smooth approximation of conic Kähler metric with lower Ricci curvature bound

\section{LIANGMING SHEN}

Maps from the enveloping algebra of the positive Witt algebra to regular algebras

Susan J. SierRa and Chelsea Walton 